arXiv version: fonts, pagination and layout may vary from GTM published version

\title{
Conormal bundles, contact homology and knot invariants
}

\author{
LENHARD NG
}

\begin{abstract}
We summarize recent work on a combinatorial knot invariant called knot contact homology. We also discuss the origins of this invariant in symplectic topology, via holomorphic curves and a conormal bundle naturally associated to the knot.
\end{abstract}

57M27; 53D40, 81T30

\section{Introduction}

String theory has provided a beautiful correspondence between enumerative geometry and knot invariants; for details, see the survey by Mariño [16] or other papers in the present volume. This correspondence applies methods from physics and algebraic geometry to a construction, described below, which is essentially symplectic.

To symplectic geometers, there is a natural way to study this same construction by counting holomorphic curves. The symplectic approach leads to a knot invariant which seems to be distinct from the invariants from physics. This manuscript serves as a survey of work by the author $[18,19,17]$ studying this invariant, which is called knot contact homology.

The string-theoretic approach originated in a conjecture of 't Hooft relating large- $N$ gauge theories and open string theories, and a subsequent observation by Witten [23] connecting Chern-Simons theory and topological strings; it was pioneered in influential papers of Gopakumar and Vafa [14] and, most importantly for our purposes, Ooguri and Vafa [20]. We will not attempt to present a careful description of the Ooguri-Vafa construction, but only an extremely brief summary.

A knot $K$ in $S^{3}$ gives rise to a natural Lagrangian submanifold $\mathcal{L} K$ in the symplectic manifold $T^{*} S^{3}$ which is the conormal bundle to $K$ :

$$
\mathcal{L} K=\left\{(x, \xi) \mid x \in K \text { and }\langle\xi, v\rangle=0 \text { for all } v \in T_{x} K\right\} \subset T^{*} S^{3}
$$

There is a way of deforming $T^{*} S^{3}$ into the manifold $\mathcal{O}(-1) \oplus \mathcal{O}(-1) \rightarrow \mathbb{P}^{1}$, by collapsing the zero section and then performing a "small resolution" of the resulting conifold singularity. This deformation then gives rise to a Lagrangian submanifold 
$\widetilde{\mathcal{L}} K \subset \mathcal{O}(-1) \oplus \mathcal{O}(-1)$ which agrees with $\mathcal{L} K \subset T^{*} S^{3}$ away from the deformation. There is then a correspondence between knot invariants of $K$ derived from Chern-Simons theory, and relative Gromov-Witten enumerative invariants of $\widetilde{\mathcal{L}} K$ :
$T^{*} S^{3}$
conifold transition
$\mathcal{O}(-1) \oplus \mathcal{O}(-1)$
$\mathcal{L} K$
$\widetilde{\mathcal{L}} K$
C-S knot invariants

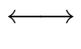
G-W holomorphic invariants

From the viewpoint of symplectic geometry, however, it is natural to look at enumerative holomorphic-curve invariants on the left side of this correspondence, before the conifold transition. This gives rise to the knot invariants which are the subject of the present paper.

We now describe how the symplectic knot invariants arise. Consider a general $n-$ dimensional Riemannian manifold $M$ with a compact submanifold $K \subset M$. The Ooguri-Vafa setup uses a knot $K$ in $M=S^{3}$; for technical reasons, it is simpler in the symplectic setup to use $M=\mathbb{R}^{3}$ instead. There is a canonical 1 -form $\lambda$ on the total space of $T^{*} M$ which pairs the result of the projections $T\left(T^{*} M\right) \rightarrow T M$ and $T\left(T^{*} M\right) \rightarrow T^{*} M$, and the natural symplectic structure on $T^{*} M$ is given by $\omega=-d \lambda$. (In local coordinates $x_{i}$ on $M$ and $\xi^{i}$ in the corresponding cotangent directions, $\lambda=\xi^{i} d x_{i}$ and $\omega=d x_{i} \wedge d \xi^{i}$.) It is easy to check that the conormal bundle $\mathcal{L} K$ is Lagrangian in $T^{*} M$; that is, $\mathcal{L} K$ has dimension $n$ and $\left.\omega\right|_{\mathcal{L} K}=0$.

Geometrically, $\mathcal{L} K$ is somewhat awkward to use, because it is noncompact. Instead, given a metric on $M$, we can work with the unit conormal bundle $L K$, which is the intersection of $\mathcal{L} K$ with the cosphere bundle $S T^{*} M=\left\{(x, \xi) \in T^{*} M \mid\|\xi\|=1\right\}$; see Figure 1. The cosphere bundle $S T^{*} M$ has a natural contact form; recall that a contact form, the odd-dimensional analogue of a symplectic form, is a 1-form $\alpha$ such that $\alpha \wedge d \alpha^{n-1}$ is nowhere zero. In this case, the contact form is given by $\alpha=\left.\lambda\right|_{S T^{*} M}$. It should be noted that the contact structure on $S T^{*} M$ induced by $\alpha$, defined to be the distribution $\operatorname{ker} \alpha$, is independent of the metric on $M$ and hence depends only on the smooth structure on $M$. With respect to this contact structure, the unit conormal $L K$ is Legendrian, meaning that $L K$ has dimension $n-1$ and $\left.\alpha\right|_{L K}=0$.

Ambient isotopy of $K$ in $M$ leads to an isotopy of $L K$ in $S T^{*} M$ through Legendrian submanifolds; hence a Legendrian-isotopy invariant of $L K$ yields a smooth-isotopy invariant of $K$, that is, a knot invariant in the case where $K$ is a knot. A Legendrianisotopy invariant in contact topology is provided by Legendrian contact homology, Eliashberg [8], which in this case is the simplest nontrivial approximation to the Symplectic Field Theory [9] of Eliashberg, Givental, and Hofer. We will define Legendrian contact homology more carefully in Section 2, but, roughly speaking, it 

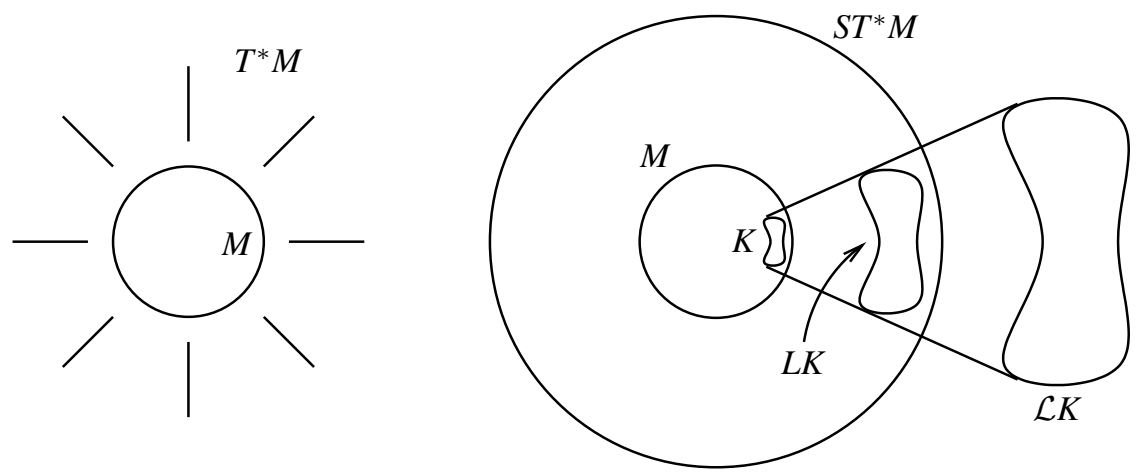

Figure 1: Schematic depiction of the cotangent bundle of $M$ (left) and the conormals $\mathcal{L} K \subset T^{*} M$ and $L K \subset S T^{*} M$ (right). Note that $\mathcal{L} K$ intersects $M$ in the knot $K$ and $S T^{*} M$ in the unit conormal $L K$.

counts holomorphic curves with boundary on a Lagrangian cylinder over the Legendrian submanifold.

The knot invariant given by Legendrian contact homology, which we call knot contact homology and denote by $H C_{*}(K)$, takes the form of a homology graded in degrees greater than or equal to 0 . We describe several combinatorial forms for knot contact homology in Section 3. For more details, see [18, 19, 17]. It is likely that knot contact homology has close ties to the "string topology" introduced by Chas and Sullivan [2].

Because of the similarity of the symplectic and string-theoretic pictures, one might expect that knot contact homology incorporates Chern-Simons knot invariants such as the Jones polynomial. Whether this is true remains to be seen, but knot contact homology is at least connected to "classical" knot invariants such as the Alexander polynomial and the A-polynomial, Cooper-Culler-Gillet-Long [5]; through the latter, knot contact homology has ties to $S L_{2} \mathbb{C}$-representations of the knot group. We discuss these relations in Section 4.

We remark that the symplectic approach produces isotopy invariants of any submanifolds in any manifold, not just knots. What these invariants might be for higher-dimensional "knots" is presently unknown.

\section{Acknowledgments}

I would like to thank Dave Auckly and Jim Bryan for organizing the very illuminating BIRS workshop "The interaction of finite type and Gromov-Witten invariants," and 
the participants of the workshop for helping me to streamline my presentation of the constructions described in this manuscript. This work was supported by an American Institute of Mathematics Five-Year Fellowship.

\section{Legendrian contact homology}

Let $V$ be a contact manifold with contact form $\alpha$, and let $L \subset V$ be a Legendrian submanifold. The Reeb vector field $R_{\alpha}$ on $V$ is uniquely defined by the conditions $\iota\left(R_{\alpha}\right) d \alpha=0, \alpha\left(R_{\alpha}\right)=1$. A Reeb orbit in $V$ is a closed orbit of the flow under the Reeb vector field, and a Reeb chord of $L$ is a path along the flow of the Reeb vector field which begins and ends on $L$. The symplectization of $V$ is the symplectic manifold $W=V \times \mathbb{R}$ with symplectic form $\omega=d\left(e^{t} \alpha\right)$, where $t$ is the coordinate on $\mathbb{R}$ and $\alpha$ is induced from $V$. We can give $W$ an almost complex structure by choosing any almost complex structure on the distribution $\operatorname{ker} \alpha$, and further setting $J \partial_{t}=R_{\alpha}$.

The Symplectic Field Theory of $V$ is a Floer-theoretic algebraic structure derived from counting holomorphic curves in the symplectization of $V$ which limit to Reeb orbits at $\pm \infty$. As a more manageable approximation, we can study Eliashberg and Hofer's contact homology [8], which counts genus-0 holomorphic curves with one end at $+\infty$ and an arbitrary number of ends at $-\infty$. More precisely, the curves in question are holomorphic maps from a multiply-punctured sphere to $V \times \mathbb{R}$ such that a neighborhood of one puncture tends to a cylinder over a Reeb orbit as $t \rightarrow+\infty$, and neighborhoods of the other punctures tend to cylinders over Reeb orbits as $t \rightarrow-\infty$. Out of the Reeb orbits and these holomorphic curves, one forms a graded complex whose homology is called contact homology and is an invariant of the contact structure.

Given a Legendrian submanifold $L \subset V$, one can construct a relative version of contact homology called Legendrian contact homology. For simplicity, we will assume that the ambient manifold $V$ has no closed Reeb orbits; this holds, for instance, in the case $V=S T^{*} \mathbb{R}^{3}$. One then counts holomorphic disks which limit to Reeb chords of $L$ at $t \rightarrow \pm \infty$, and whose boundary lies on $L$.

More precisely, suppose that $L$ has a finite number of Reeb chords, which we label $a_{1}, \ldots, a_{n}$. The complex whose homology yields Legendrian contact homology is the tensor algebra $\mathcal{A}$ freely generated by $a_{1}, \ldots, a_{n}$ over the group ring $\mathbb{Z}\left[H_{1}(L)\right]$; that is, $\mathcal{A}$ is the module over $\mathbb{Z}\left[H_{1}(L)\right]$ generated by (noncommutative) words in $a_{1}, \ldots, a_{n}$, including the empty word. This algebra can be given a grading by placing the base ring $\mathbb{Z}\left[H_{1}(L)\right]$ in degree 0 , and assigning degrees to the generators $a_{i}$ which are essentially 

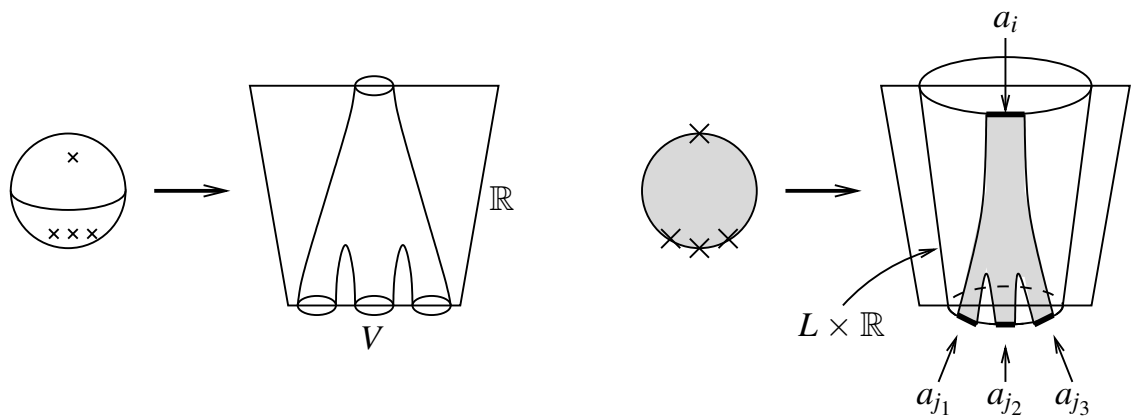

Figure 2: On the left, a holomorphic curve in $V \times \mathbb{R}$ contributing to the contact homology of $V$. The punctures are mapped to the cylindrical ends, which approach cylinders over Reeb orbits of $V$. On the right, a holomorphic disk in $V \times \mathbb{R}$ contributing to the Legendrian contact homology of $L$. Here the boundary of the disk, away from the punctures, is mapped to $L \times \mathbb{R}$, and neighborhoods of the punctures are mapped to strips approaching Reeb chords of $L$. This disk contributes the term $a_{j_{1}} a_{j_{2}} a_{j_{3}}$ to $\partial a_{i}$.

the Conley-Zehnder indices of the Reeb chords; see [7]. In order to define a differential on $\mathcal{A}$, we need to study certain moduli spaces of holomorphic curves.

For each Reeb chord $a_{i}$, choose a "capping path" lying in $L$ which joins the endpoints of the chord. Let $D_{k}$ be a disk with boundary, minus boundary punctures $x, y_{1}, \ldots, y_{k}$ appearing in order around the boundary, and give $D_{k}$ the complex structure induced from the unit disk in $\mathbb{C}$. For $A \in H_{1}(L)$, we define the moduli space $\mathcal{M}^{A}\left(a_{i} ; a_{j_{1}}, \ldots, a_{j_{k}}\right)$ to be the set of maps $f: D_{k} \rightarrow V \times \mathbb{R}$ such that:

- $f$ is holomorphic and proper and has finite energy with respect to $d \alpha$;

- $f$ maps the boundary of $D_{k}$ (without the punctures) to $L \times \mathbb{R}$;

- $f$ maps a neighborhood of the puncture $x$ to a strip approaching the strip $a_{i} \times \mathbb{R}$ as $t \rightarrow+\infty$;

- $f$ maps a neighborhood of the puncture $y_{i}$ to a strip approaching the strip $a_{j_{i}} \times \mathbb{R}$ as $t \rightarrow-\infty$;

- the image of the boundary of $D_{k}$, made into a closed curve by appending the appropriate capping paths, is in the homology class $A$.

Note that there is an $\mathbb{R}$-action on $\mathcal{M}^{A}\left(a_{i} ; a_{j_{1}}, \ldots, a_{j_{k}}\right)$ given by translation in the $\mathbb{R}$ direction. The moduli spaces that interest us are the ones that are rigid modulo this $\mathbb{R}$-action

Define the differential of $a_{i}$ as

$$
\partial a_{i}=\sum_{\operatorname{dim} \mathcal{M}^{A}\left(a_{i} ; a_{j_{1}}, \ldots, a_{j_{k}}\right)=1} \#\left(\mathcal{M}^{A}\left(a_{i} ; a_{j_{1}}, \ldots, a_{j_{k}}\right) / \mathbb{R}\right) A a_{j_{1}} \ldots a_{j_{k}},
$$


where $\#\left(\mathcal{M}^{A}\left(a_{i} ; a_{j_{1}}, \ldots, a_{j_{k}}\right) / \mathbb{R}\right)$ is the signed number of points in the 0 -dimensional space $\mathcal{M} / \mathbb{R}$; extend $\partial$ to $\mathcal{A}$ via the Leibniz rule. Then $(\mathcal{A}, \partial)$ becomes a differential graded algebra, usually abbreviated in the subject as a DGA.

Theorem 2.1 $\partial^{2}=0, \partial$ lowers degree by 1 , and the graded homology $H_{*}(\mathcal{A}, \partial)$ is an invariant of the Legendrian isotopy class of $L$.

It is currently a bit of a misnomer to label this result, in full generality, as a theorem, since the analytical details to prove it are still being worked out. The foundational analysis has been performed in several cases of interest, including $\mathbb{R}^{3}[10], \mathbb{R}^{2 n+1}$ for $n>1$ [7], and jet spaces. On the other hand, there is a standard technique for sidestepping these analytical issues by finding a purely combinatorial form for the differential graded algebra $(\mathcal{A}, \partial)$ and proving that this combinatorial algebra is invariant under Legendrian isotopy. This technique dates back to Chekanov's pioneering work [3] on Legendrian knots in standard contact $\mathbb{R}^{3}$, and is the strategy used in $[18,19,17]$ to define knot contact homology.

In the case of interest here, $V$ is the contact manifold $S T^{*} \mathbb{R}^{3}=\mathbb{R}^{3} \times S^{2}$, which can also be viewed as the 1 -jet space $J^{1}\left(S^{2}\right)$, and $L$ is the Legendrian torus $L K$ given by the unit conormal to the knot $K$ in $S T^{*} \mathbb{R}^{3}$. Given a framing and an orientation of $K$, we obtain longitude and meridian classes $\lambda, \mu \in H_{1}(L K)$ and hence an identification of $\mathbb{Z}\left[H_{1}(L K)\right]$ with $\mathbb{Z}\left[\lambda^{ \pm 1}, \mu^{ \pm 1}\right]$.

The Reeb vector field lies within the $\mathbb{R}^{3}$ fibers in $\mathbb{R}^{3} \times S^{2}$; in the fiber lying over $\xi \in S^{2}$, it simply points in the direction of the dual to $\xi$. It follows that Reeb chords of $L K$ correspond to "binormal chords": oriented line segments beginning and ending on $K$ which are normal to $K$ at both endpoints. If we parametrize $K$ by $S^{1}$, then the distance function in $\mathbb{R}^{3}$ between points on $K$ gives a map $d: S^{1} \times S^{1} \rightarrow \mathbb{R}$ whose nondiagonal critical points are binormal chords. One can then set up the theory so that the degree of a Reeb chord in the contact homology DGA is the Morse index of the corresponding critical point of $d$. Hence the generators of the DGA all have degree 0,1 , or 2 ; it follows that the DGA is a nonnegatively graded algebra over the ring $\mathbb{Z}\left[\lambda^{ \pm 1}, \mu^{ \pm 1}\right]$.

We can view Legendrian contact homology in $S T^{*} \mathbb{R}^{3}$ as counting curves in $T^{*} \mathbb{R}^{3}$. The symplectization $S T^{*} \mathbb{R}^{3} \times \mathbb{R}$ is symplectically diffeomorphic to $T^{*} \mathbb{R}^{3} \backslash \mathbb{R}^{3}$, the cotangent bundle minus the zero section, via the map sending $((x, \xi), t)$ to $\left(x, e^{t} \xi\right)$. The $t \rightarrow+\infty$ end of the symplectization maps to the unbounded "end" of $T^{*} \mathbb{R}^{3}$, while the $t \rightarrow-\infty$ end maps to the zero section. Under this identification, the holomorphic curves used to calculate Legendrian contact homology are maps $f$ of boundary-punctured disks to $T^{*} \mathbb{R}^{3} \backslash \mathbb{R}^{3}$ satisfying the following properties: 


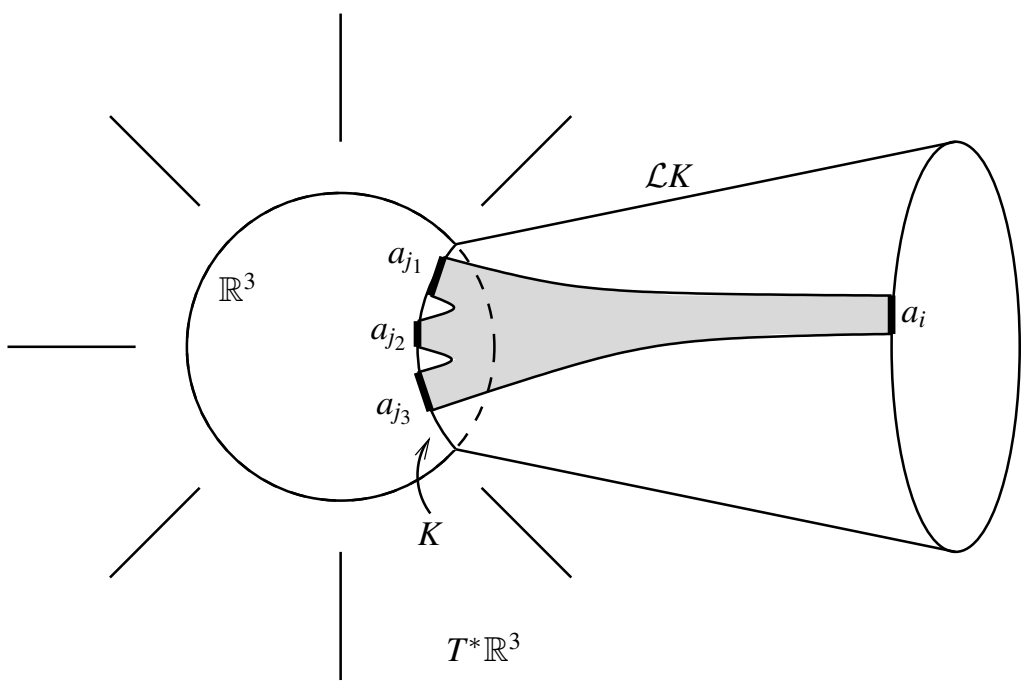

Figure 3: Holomorphic disk used in the computation of the Legendrian contact homology of $L K$. The ambient space is $T^{*} \mathbb{R}^{3}$. In this picture, the holomorphic disk has boundary on $\mathcal{L} K$, except for the strips which limit to the binormal chords $a_{j_{1}}, a_{j_{2}}, a_{j_{3}}$ on the zero section and the binormal chord $a_{i}$ at infinity.

- $f$ is holomorphic, proper, and finite energy;

- $f$ sends the boundary of the disk to $\mathcal{L} K$;

- $f$ sends a neighborhood of one puncture to a strip which approaches, on the unbounded end of $T^{*} \mathbb{R}^{3}$ (in the fiber direction), a cylindrical strip of the form $B C \times\left\{e^{t} \xi \mid t \gg 0\right\} \subset T^{*} \mathbb{R}^{3}$, where $B C$ is a binormal chord and $\xi$ is a covector dual to the direction of $B C$;

- $f$ sends neighborhoods of the other punctures to neighborhoods of binormal chords of $K$ in the zero section of $T^{*} \mathbb{R}^{3}$.

See Figure 3.

We remark that the curves which produce Legendrian contact homology in this context are somewhat different from the curves which would be counted in relative GromovWitten theory; it is unknown what relation our invariant has to the Chern-Simons type invariants studied by Ooguri-Vafa et al. Also note that the almost complex structure on $T^{*} \mathbb{R}^{3} \backslash \mathbb{R}^{3}$ inherited from the symplectization, which we use to define our holomorphic curves, cannot be extended to all of $T^{*} \mathbb{R}^{3}$.

This discussion of Legendrian contact homology should be viewed as motivation for the knot contact homology invariant which will be defined combinatorially in the following 
section. There is work in progress to verify that the combinatorial DGA actually yields Legendrian contact homology, by examining gradient flow trees on the front of the Legendrian torus $L K$, and using the relation between gradient trees and holomorphic curves described by Fukaya and Oh [12]. As mentioned earlier, one can instead directly show that the combinatorial theory gives a topological knot invariant, without using its origin in symplectic geometry; this is the approach of $[18,19,17]$, and will be our approach in the next section.

\section{Knot contact homology: definition}

Let $K$ be an oriented knot in $\mathbb{R}^{3}$; this has a canonical framing given by any Seifert surface. As discussed in the previous section, the Legendrian contact homology of $L K \subset S T^{*} \mathbb{R}^{3}$, which is a knot invariant, is the homology of a complex defined in nonnegative degree over the ring $\mathbb{Z}\left[\lambda^{ \pm 1}, \mu^{ \pm 1}\right]$. We will now give a combinatorial definition of this complex, which is called the framed knot DGA. (This complex may be different from the one described in the previous section, but it has the same homology.)

Fix a knot diagram for $K$ with $n$ crossings. The framed knot DGA is the algebra $\mathcal{A}$ over $\mathbb{Z}\left[\lambda^{ \pm 1}, \mu^{ \pm 1}\right]$ freely generated by the following generators:

- $\left\{a_{i j}\right\}_{1 \leq i, j \leq n, i \neq j}$ of degree 0 ;

- $\left\{b_{\alpha i}\right\}_{1 \leq \alpha, i \leq n}$ and $\left\{c_{i \alpha}\right\}_{1 \leq \alpha, i \leq n}$ of degree 1;

- $\left\{d_{\alpha \beta}\right\}_{1 \leq \alpha, \beta \leq n}$ and $\left\{e_{\alpha}\right\}_{1 \leq \alpha \leq n}$ of degree 2 .

Here Greek subscripts represent crossings in the knot diagram, numbered arbitrarily from 1 to $n$, and Roman subscripts represent components of the knot diagram, from undercrossing to undercrossing, also numbered arbitrarily from 1 to $n$.

To define the differential on $\mathcal{A}$, we first need some auxiliary definitions. Each crossing $i$ involves three components of the knot diagram, the overstrand $o_{i}$ and the understrands $l_{i}$ and $r_{i}$, distinguished as being on the left and right sides as the overstrand is traversed in the direction of the knot's orientation. Define $\epsilon_{1}$ to be \pm 1 depending on the sign of crossing 1 , where sign is defined in terms of the knot's orientation in the usual way. (If $l_{1}$ follows $r_{1}$ when traversing the knot, then $\epsilon_{1}=1$; if $r_{1}$ follows $l_{1}$, then $\epsilon_{1}=-1$.) 
Let $\Psi^{L}, \Psi^{R}, \Psi_{2}^{L}, \Psi_{1}^{R}$ be the $n \times n$ matrices defined as follows:

$$
\begin{aligned}
& \left(\Psi^{L}\right)_{\alpha i}=\left\{\begin{array}{ll}
\lambda^{-\epsilon_{1}} & \alpha=1, i=r_{1} \\
1 & \alpha \neq 1, i=r_{\alpha} \\
\mu & i=l_{\alpha} \\
-a_{l_{\alpha} o_{\alpha}} & i=o_{\alpha} \\
0 & \text { otherwise }
\end{array} \quad\left(\Psi^{R}\right)_{i \alpha}= \begin{cases}\lambda^{\epsilon_{1}} \mu & \alpha=1, i=r_{1} \\
\mu & \alpha \neq 1, i=r_{\alpha} \\
1 & i=l_{\alpha} \\
-a_{o_{\alpha} l_{\alpha}} & i=o_{\alpha} \\
0 & \text { otherwise }\end{cases} \right. \\
& \left(\Psi_{2}^{L}\right)_{\alpha i}=\left\{\begin{array}{ll}
\mu & i=l_{\alpha} \\
-a_{l_{\alpha} o_{\alpha}} & i=o_{\alpha} \\
0 & \text { otherwise }
\end{array} \quad\left(\Psi_{1}^{R}\right)_{i \alpha}= \begin{cases}\lambda^{\epsilon_{1}} \mu & \alpha=1, i=r_{1} \\
\mu & \alpha \neq 1, i=r_{\alpha} \\
0 & \text { otherwise. }\end{cases} \right.
\end{aligned}
$$

Assemble generators of $\mathcal{A}$ into $n \times n$ matrices $A, B, C, D$ as follows:

$$
A_{i j}=\left\{\begin{array}{rl}
1+\mu & \text { if } i=j \\
a_{i j} & \text { if } i \neq j
\end{array} ; \quad B_{\alpha i}=b_{\alpha i} ; \quad C_{i \alpha}=c_{i \alpha} ; \quad \text { and } D_{\alpha \beta}=d_{\alpha \beta} .\right.
$$

Write $\partial A$ for the matrix whose entries are the differentials of the corresponding entries of $A$, and similarly for $\partial B, \partial C$, and $\partial D$. Then the differential on generators is given by

$$
\begin{aligned}
\partial A & =0 \\
\partial B & =\Psi^{L} \cdot A \\
\partial C & =A \cdot \Psi^{R} \\
\partial D & =B \cdot \Psi^{R}-\Psi^{L} \cdot C \\
\partial e_{\alpha} & =\left(B \cdot \Psi_{1}^{R}-\Psi_{2}^{L} \cdot C\right)_{\alpha \alpha} .
\end{aligned}
$$

Extend this via the Leibniz rule to obtain a differential on all of $\mathcal{A}$.

Proposition 3.1 [17] $\partial^{2}=0$ and the homology $H C_{*}(K)=H_{*}(\mathcal{A}, \partial)$ depends only on the isotopy class of $K$, not the particular knot diagram. This homology is called the (framed) knot contact homology of $K$.

There is an equivalence relation on semifree differential graded algebras known as stable tame isomorphism [3], under which $(\mathcal{A}, \partial)$ is independent of the knot diagram; equivalent DGAs have isomorphic homology. Up to equivalence, we can refer to $(\mathcal{A}, \partial)$ as the framed knot DGA of $K$. For a proof of invariance, using a somewhat different formulation of the framed knot DGA, see [17].

The above definition of the framed knot DGA is combinatorial and relatively simple, but opaque. A purely topological interpretation for the full DGA or its homology would be very interesting but is presently lacking. Most current applications of knot contact 
homology use only its lowest-degree component, the degree-0 homology $H C_{0}(K)$, which does have a topological formulation [19] as we now describe.

Let $K \subset \mathbb{R}^{3}=S^{3} \backslash\{\mathrm{pt}\}$ be an oriented knot equipped with the zero framing, and let $l, m$ denote the homotopy classes of the longitude and meridian of $K$ in $\pi_{1}\left(S^{3} \backslash K\right)$. Let $\mathcal{A}_{K}$ denote the tensor algebra over $\mathbb{Z}\left[\lambda^{ \pm 1}, \mu^{ \pm 1}\right]$ freely generated by the set $\pi_{1}\left(S^{3} \backslash K\right)$; a monomial in $\mathcal{A}_{K}$ looks like $\left[\gamma_{1}\right]\left[\gamma_{2}\right] \ldots\left[\gamma_{k}\right]$, where $\left[\gamma_{i}\right]$ denotes the image of $\gamma_{i} \in \pi_{1}\left(S^{3} \backslash K\right)$ in $\mathcal{A}_{K}$.

Definition 3.2 The cord algebra of $K$ is the quotient of $\mathcal{A}_{K}$ by the relations

- $[e]=1+\mu$;

- $[\gamma m]=[m \gamma]=\mu[\gamma]$ and $[\gamma l]=[l \gamma]=\lambda[\gamma]$ for $\gamma \in \pi_{1}\left(S^{3} \backslash K\right)$;

- $\left[\gamma_{1} \gamma_{2}\right]+\left[\gamma_{1} m \gamma_{2}\right]=\left[\gamma_{1}\right]\left[\gamma_{2}\right]$ for $\gamma_{1}, \gamma_{2} \in \pi_{1}\left(S^{3} \backslash K\right)$.

If $K$ is the unknot, then $\pi_{1}\left(S^{3} \backslash K\right) \cong \mathbb{Z}$ is generated by $m$, and the above relations yield $[e]=1+\mu$ and $[l]=[e]=\lambda[e]$; it follows easily that the cord algebra of the unknot is $\mathbb{Z}\left[\lambda^{ \pm 1}, \mu^{ \pm 1}\right] /((\lambda-1)(\mu+1))$.

For general knots, the cord algebra is evidently a knot invariant, but is difficult to compute using Definition 3.2 directly. On the other hand, degree-0 knot contact homology is readily computable in terms of generators and relations, but is not obviously a topological invariant. A key result in [17] (see also [19]) states that these two constructions coincide; we will discuss the intuitive explanation for this result later in the section.

Proposition 3.3 $[19,17]$ The cord algebra of $K$ is isomorphic to $H C_{0}(K)$. Given an $n$-crossing knot diagram of $K$, the cord algebra can be expressed as the tensor algebra $\mathcal{A}$ over $\mathbb{Z}\left[\lambda^{ \pm 1}, \mu^{ \pm 1}\right]$ generated by $\left\{a_{i j}\right\}_{1 \leq i, j \leq n, i \neq j}$ modulo the $2 n^{2}$ relations given by the entries of the matrices $\Psi^{L} \cdot A$ and $A \cdot \Psi^{R}$.

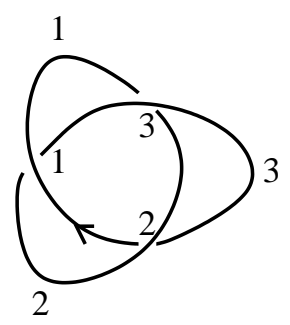

Figure 4: The left-handed trefoil, with crossings and diagram components labeled. 
Suppose, for instance, that we wish to compute the cord algebra of the left-handed trefoil shown in Figure 4. We have

$$
\Psi^{L}=\left(\begin{array}{ccc}
-a_{21} & \mu & \lambda \\
1 & -a_{32} & \mu \\
\mu & 1 & -a_{13}
\end{array}\right), \quad \Psi^{R}=\left(\begin{array}{ccc}
-a_{12} & \mu & 1 \\
1 & -a_{23} & \mu \\
\lambda^{-1} \mu & 1 & -a_{31}
\end{array}\right), \quad \text { and } A=\left(\begin{array}{ccc}
1+\mu & a_{12} & a_{13} \\
a_{21} & 1+\mu & a_{23} \\
a_{31} & a_{32} & 1+\mu
\end{array}\right) .
$$

When we equate the entries of $\Psi^{L} \cdot A$ and $A \cdot \Psi^{R}$ to zero, we find that $a_{31}=\lambda^{-1} a_{21}$, $a_{13}=\lambda a_{12}, a_{32}=a_{12}, a_{23}=a_{21}$, and $a_{23}=a_{13}$; it follows that we can substitute for everything in terms of $a_{12}$. If we write $x=a_{12}$, then the relations reduce to two polynomials in $x$, and the cord algebra becomes

$$
H C_{0}(\mathrm{LH} \text { trefoil }) \cong \mathbb{Z}\left[\lambda^{ \pm 1}, \mu^{ \pm 1}\right][x] /\left(\lambda x^{2}-\lambda x-\mu^{2}-\mu, \lambda x^{2}-\mu x-\mu-1\right) .
$$

This is different from the cord algebra of the unknot (set $\lambda=\mu=1$ ), and also from the cord algebra of the right hand trefoil. Section 4 discusses general methods for distinguishing knot contact homologies and relations to classical invariants.

The term "cord algebra" comes from another interpretation of $H C_{0}(K)$. Fix a point * on the knot $K$. A cord of $K$ (terminology due to D. Bar-Natan) is a path in $S^{3}$ which begins and ends on $K \backslash\{*\}$ and does not intersect $K$ in the interior of the path. Let $\widetilde{\mathcal{A}}_{K}$ denote the tensor algebra over $\mathbb{Z}\left[\lambda^{ \pm 1}, \mu^{ \pm 1}\right]$ generated by homotopy classes of cords. In $\widetilde{\mathcal{A}}_{K}$, mod out by several relations:

- the contractible homotopy class is equal to $1+\mu$;

- if two cords are related by pulling an endpoint along $K$ across $*$, then one is $\lambda$ times the other (the precise choice is given by orientations);

- if two cords are related by pushing an interior point in the cord through $K$, then at the moment when the interior point crosses $K$, the cord breaks into two, and the four cords involved are related by

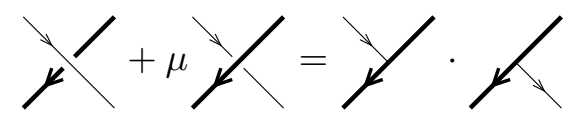

where the knot is drawn thickly and the cords thinly.

Then $\widetilde{\mathcal{A}}_{K}$ modulo these relations is isomorphic to the cord algebra of $K$. To illustrate this isomorphism, push the endpoints of any cord slightly off of the knot, and join the endpoints via a path running parallel to the knot, to obtain a closed curve in $S^{3} \backslash K$. By carefully tracking the various choices involved, we can map cords to elements of $\pi_{1}\left(S^{3} \backslash K\right)$, and the relations imposed on cords become the relations in Definition 3.2.

The isomorphism from Proposition 3.3 between the cord algebra and $H C_{0}(K)$ can now be seen as follows. Place the point $*$ at the undercrossing of crossing number 1 , and 
send to $a_{i j}$ the cord which begins on strand $i$, ends on strand $j$, and lies strictly above the plane of the diagram in between. The relations $\Psi^{L} \cdot A$ and $A \cdot \Psi^{R}$ which define $H C_{0}(K)$ are simply the result of applying the cord "skein relation" above when an endpoint of the cord $a_{i j}$ is pulled through a crossing. See [17] for details.

Cords also give us a heuristic way to see the correspondence between the cord algebra $H C_{0}(K)$ and the abstract version of knot contact homology described in Section 2. If we imagine a cord as a rubber band and pull it tight, then we can use the cord skein relation above to write any cord in terms of minimal binormal chords, that is, line segments which locally minimize distance between points on the knot. On the other hand, since the DGA which produces knot contact homology has generators only in nonnegative degree, any chain in degree 0 is a cycle; hence the degree- 0 homology is a quotient of the algebra generated by the degree- 0 Reeb chords, which are precisely minimal binormal chords.

The results described in this section, and particularly the interpretation of $H C_{0}$ as an algebra of cords, can be viewed as a relative analogue of the work of Viterbo [22], Salamon and Weber [21], and Abbondandolo and Schwarz [1], which shows, roughly speaking, that the symplectic Floer homology of the cotangent bundle of a manifold is given by the homology of the loop space of the manifold. In our case, there is a relation between a relative Floer theory connected to the cotangent bundle and an algebra generated by "relative loops" with endpoints on the knot. It is likely in this context that knot contact homology is related to string topology [2]; see [4] for more on the possible relation in the absolute case.

\section{Knot contact homology: properties}

Knot contact homology, and in particular the cord algebra $H C_{0}(K)$, is a reasonably strong knot invariant.

Proposition 4.1 $[18,17]$ As an algebra over $\mathbb{Z}\left[\lambda^{ \pm 1}, \mu^{ \pm 1}\right], H C_{0}(K)$ can distinguish between mirrors and also between Conway mutants. There are examples where the

cord algebra is stronger than any one of the following invariants: Alexander polynomial, Jones polynomial, HOMFLY polynomial, Kauffman polynomial, Khovanov homology, Ozsváth-Szabó invariant.

It is even conceivable at present that the cord algebra could be a complete knot invariant. 
Although the cord algebra is often a difficult object to handle directly, one can easily compute numerical invariants from it known as augmentation numbers, and Proposition 4.1 is proven using these numbers. Given a $\operatorname{ring} R$, which one usually takes to be a finite field $\mathbb{Z}_{p}$, augmentation numbers count the number of maps which send $H C_{0}(K)$ to $R$, given particular choices of invertible elements in $R$ for the images of $\lambda$ and $\mu$. The set of possible maps is finite for given $p$ and can be computed using a program such as Mathematica; the relevant code can be found on the author's web page.

Knot contact homology has a couple of direct relations to "classical" knot invariants.

Proposition 4.2 [17] One can deduce the Alexander invariant, and thus the Alexander polynomial, from a linearized form of the framed knot DGA with $\lambda=1$.

It is also likely that one can find the Alexander invariant in the cord algebra $H C_{0}(K)$, without needing to use higher degree contact homology.

A more interesting link is that between the cord algebra and the $A$-polynomial of [5]. The $A$-polynomial is defined in terms of $S L_{2} \mathbb{C}$ representations of the knot group, essentially as follows. For an oriented knot $K$ in $S^{3}$, the knot group $\pi_{1}\left(S^{3} \backslash K\right)$ has two distinguished elements, the longitude $l$ and meridian $m$ of $K$. Since these two elements commute, any representation $\rho: \pi_{1}\left(S^{3} \backslash K\right) \rightarrow S L_{2} \mathbb{C}$ is conjugate to one in which $l$ and $m$ are sent to upper triangular (typically diagonal) matrices

$$
\rho(l)=\left(\begin{array}{cc}
\lambda & * \\
0 & \lambda^{-1}
\end{array}\right) \quad \text { and } \quad \rho(m)=\left(\begin{array}{cc}
\mu & * \\
0 & \mu^{-1}
\end{array}\right) .
$$

The set of pairs of eigenvalues $(\lambda, \mu)$ over all representations forms a variety in $\left(\mathbb{C}^{*}\right)^{2}$ whose 1-dimensional components are the zero set of the $A$-polynomial $A_{K}(\lambda, \mu)$.

One can also form a two-variable polynomial from the cord algebra. The set of pairs $(\lambda, \mu)$ in $\left(\mathbb{C}^{*}\right)^{2}$ for which there is a map $H C_{0}(K) \otimes \mathbb{C} \rightarrow \mathbb{C}$ extending the identity on $\mathbb{C}$ forms a variety, typically (perhaps always) 1 -dimensional; if it is 1 -dimensional, then it is the vanishing set of a polynomial $\widetilde{A}_{K}(\lambda, \mu)$ which we call the augmentation polynomial. For instance, the left-handed trefoil has augmentation polynomial given by the resultant of the polynomials $\lambda x^{2}-\lambda x-\mu^{2}-\mu$ and $\lambda x^{2}-\mu x-\mu-1$ which determine the cord algebra: up to units, we have

$$
\widetilde{A}_{\mathrm{LH} \text { trefoil }}(\lambda, \mu)=(\lambda-1)(\mu+1)\left(\lambda-\mu^{3}\right) .
$$

By contrast, the right-handed trefoil has augmentation polynomial

$$
\widetilde{A}_{\mathrm{RH} \text { trefoil }}(\lambda, \mu)=(\lambda-1)(\mu+1)\left(1-\lambda \mu^{3}\right) ;
$$

this shows that the cord algebra distinguishes mirrors.

The $A$-polynomial is contained in the augmentation polynomial: 
Proposition 4.3 [17] The polynomial $\left(1-\mu^{2}\right) A_{K}(\lambda, \mu)$ divides $\widetilde{A}_{K}\left(\lambda,-\mu^{2}\right)$.

Sketch of proof If we set $\mu=-1$, there is a trivial map from $H C_{0}(K)$ to $\mathbb{C}$ which sends all $[\gamma]$ 's to 0 ; it follows that $1-\mu^{2}$ divides $\widetilde{A}_{K}\left(\lambda,-\mu^{2}\right)$. Now given a representation $\rho: \pi_{1}\left(S^{3} \backslash K\right) \rightarrow S L_{2} \mathbb{C}$ such that $\rho(l)$ and $\rho(m)$ are diagonal, define $\{\gamma\} \in \mathbb{C}$, for any $\gamma \in \pi_{1}\left(S^{3} \backslash K\right)$, to be the upper left entry of $\rho(\gamma)$. Then $A_{K}(\lambda, \mu)=0$, where $\lambda=\{l\}$ and $\mu=\{m\}$, and

$$
\left\{A_{1} A_{2}\right\}-\mu\left\{A_{1} \rho(m) A_{2}\right\}=\left(1-\mu^{2}\right)\left\{A_{1}\right\}\left\{A_{2}\right\}
$$

for any $A_{1}, A_{2} \in S L_{2} \mathbb{C}$. It follows easily from the homotopy definition of the cord algebra (Definition 3.2) that the map $[\gamma] \mapsto\left(1-\mu^{2}\right)(-\mu)^{1 \mathrm{k}(\gamma, K)}\{\rho(\gamma)\}$ induces a map from $H C_{0}\left(K, \lambda,-\mu^{2}\right)$ to $\mathbb{C}$, where $H C_{0}\left(K, \lambda,-\mu^{2}\right)$ denotes the cord algebra with $\mu$ replaced by $-\mu^{2}$. Hence $\widetilde{A}_{K}\left(\lambda,-\mu^{2}\right)=0$, so $A_{K}(\lambda, \mu)$ divides $\widetilde{A}_{K}\left(\lambda,-\mu^{2}\right)$.

A recent result of Dunfield and Garoufalidis [6], building on the proof of Property $\mathrm{P}$ for knots by Kronheimer and Mrowka [15], states that nontrivial knots have nontrivial $A$-polynomial. The following result is then a direct consequence of Proposition 4.3.

Corollary 4.4 [17] Knot contact homology, or in particular the cord algebra, distinguishes the unknot.

We believe that the $A$-polynomial and augmentation polynomial coincide for two-bridge knots-more precisely, that $\widetilde{A}_{K}\left(\lambda,-\mu^{2}\right)=\left(1-\mu^{2}\right) A_{K}(\lambda, \mu)$ in this case. This is not, however, true in general. For instance, for the torus knot $T(3,4)$, we have

$$
\widetilde{A}_{T(3,4)}\left(\lambda,-\mu^{2}\right)=\left(1-\lambda \mu^{8}\right)\left(1-\mu^{2}\right) A_{T(3,4)}(\lambda, \mu) .
$$

It is possible that the augmentation polynomial contains more, or different, information than the $A$-polynomial.

Since the $A$-polynomial has links to the Jones polynomial (see, for example, [11, 13]), it is possible that knot contact homology encodes the Jones polynomial or other classical invariants. This would make sense given the relation between Chern-Simons knot invariants and holomorphic curves discussed in the introduction. At present, however, it is not known how knot contact homology might contain the Jones polynomial.

\section{References}

[1] A Abbondandolo, M Schwarz, On the Floer homology of cotangent bundles, Comm. Pure Appl. Math. 59 (2006) 254-316 
[2] M Chas, D Sullivan, String topology arXiv:math.GT/9911159

[3] Y Chekanov, Differential algebra of Legendrian links, Invent. Math. 150 (2002) 441-483 MR1946550

[4] R Cohen, Morse theory, graphs, and string topology, from: "Morse Theoretic Methods in Nonlinear Analysis and in Symplectic Topology", NATO Sci. Ser. II Math. Phys. Chem. 217, Kluwer (2006) arXiv:math.GT/0411272

[5] D Cooper, M Culler, H Gillet, D D Long, P B Shalen, Plane curves associated to character varieties of 3-manifolds, Invent. Math. 118 (1994) 47-84 MR1288467

[6] N Dunfield, S Garoufalidis, Non-triviality of the A-polynomial for knots in $S^{3}$, Algebr. Geom. Topol. 4 (2004) 1145-1153 MR2113900

[7] T Ekholm, J Etnyre, M Sullivan, The contact homology of Legendrian submanifolds in $\mathbb{R}^{2 n+1}$, J. Diff. Geom. (to appear)

[8] Y Eliashberg, Invariants in contact topology, Doc. Math. (1998) 327-338 MR1648083

[9] Y Eliashberg, A Givental, H Hofer, Introduction to symplectic field theory, Geom. Funct. Anal. (2000) 560-673 MR1826267

[10] J B Etnyre, L L Ng, J M Sabloff, Invariants of Legendrian knots and coherent orientations, J. Symplectic Geom. 1 (2002) 321-367 MR1959585

[11] C Frohman, R Gelca, W Lofaro, The A-polynomial from the noncommutative viewpoint, Trans. Amer. Math. Soc. 354 (2002) 735-747 MR1862565

[12] K Fukaya, Y-G Oh, Zero-loop open strings in the cotangent bundle and Morse homotopy, Asian J. Math. 1 (1997) 96-180 MR1480992

[13] S Garoufalidis, On the characteristic and deformation varieties of a knot, from: "Proceedings of the Casson Fest", Geom. Topol. Monogr. 7, Geom. Topol. Publ., Coventry, UK (2004) 291-309

[14] R Gopakumar, C Vafa, On the gauge theory/geometry correspondence, Adv. Theor. Math. Phys. 3 (1999) 1415-1443 MR1796682

[15] P B Kronheimer, TS Mrowka, Dehn surgery, the fundamental group and SU(2), Math. Res. Lett. 11 (2004) 741-754 MR2106239

[16] M Mariño, Enumerative geometry and knot invariants, from: "Infinite dimensional groups and manifolds", IRMA Lect. Math. Theor. Phys. 5, de Gruyter, Berlin (2004) 27-92 MR2104354

[17] L Ng, Framed knot contact homology arXiv:math.GT/0407071

[18] L Ng, Knot and braid invariants from contact homology I, Geom. Topol. 9 (2005) 247-297 MR2116316

[19] L Ng, Knot and braid invariants from contact homology II, Geom. Topol. 9 (2005) 1603-1637

[20] H Ooguri, C Vafa, Knot invariants and topological strings, Nuclear Phys. B 577 (2000) 419-438 MR1765411 
[21] D A Salamon, J Weber, Floer homology and the heat flow, Geom. Funct. Anal. (to appear) arXiv:math.SG/0304383

[22] C Viterbo, Functors and computations in Floer homology with applications II, Geom. Funct. Anal. (to appear)

[23] E Witten, Chern-Simons gauge theory as a string theory, from: "The Floer memorial volume”, Progr. Math. 133, Birkhäuser, Basel (1995) 637-678 MR1362846

Department of Mathematics, Stanford University

Stanford, CA 94305, USA

lng@math.stanford.edu

http://alum.mit.edu/www/ng/

Received: 23 December 2004 Revised: 2 February 2005

Geometry \& Topology Monographs 8 (2006) 